\title{
Effect of solvent type and ratio on betacyanins and antioxidant activity of extracts from Hylocereus polyrhizus flesh and peel by supercritical fluid extraction and solvent extraction
}

\begin{abstract}
The main objective of the present study was to investigate the effect of solvent type and ratio as well as the extraction techniques (i.e. supercritical fluid extraction (SFE) and conventional solvent extraction) on betacyanins and antioxidant activity of the peel and fresh extract from the red pitaya (Hylocereus polyrhizus). The peel and flesh extracts obtained by SFE at 25 $\mathrm{MPa}$ pressure and 10\% EtOH/water (v/v) mixture as a co-solvent contained 24.58 and 91.27 $\mathrm{mg} / 100 \mathrm{ml}$ total betacyanin, respectively; while the most desirable solvent extraction process resulted in a relatively higher total betacyanin in the peel and flesh extracts (28.44 and 120.28 $\mathrm{mg} / 100 \mathrm{ml}$, respectively). The major betacyanins identified in the pitaya peel and flesh extracts were betanin, isobetanin, phyllocactin, butyrylbetanin, isophyllocactin and isobutyrylbetanin. The flesh extract had the stronger antioxidant activity than the peel extract when the higher proportion of ethanol to water (E/W) was applied for the extraction.
\end{abstract}

Keyword: Red pitaya; Hylocereus polyrhizus; Betacyanins; Supercritical fluid extraction; Antioxidant activity; Solvent extraction 